\title{
Liver resection for multifocal hepatocellular carcinoma: is it an option?
}

\author{
Luca Viganò $^{1,3}$, Guido Costa $^{1}$, Luca Di Tommaso ${ }^{2,3}$ \\ ${ }^{1}$ Division of Hepatobiliary \& General Surgery, Department of Surgery, ${ }^{2}$ Pathology Unit, Humanitas Clinical and Research Center, IRCCS, Rozzano, \\ Milan, Italy; ${ }^{3}$ Department of Biomedical Sciences, Humanitas University, Rozzano, Milan, Italy \\ Correspondence to: Luca Viganò, MD, PhD. Division of Hepatobiliary \& General Surgery, Department of Surgery, Humanitas Clinical and Research \\ Center, IRCCS, Humanitas University, Via A. Manzoni, 56, 20089 Rozzano, Milano, Italy. Email: luca.vigano@hunimed.eu.
}

Submitted May 08, 2019. Accepted for publication May 16, 2019.

doi: $10.21037 /$ hbsn.2019.05.12

View this article at: http://dx.doi.org/10.21037/hbsn.2019.05.12

Hepatocellular carcinoma (HCC) is the second leading cause of cancer-related death worldwide. At first diagnosis, HCC is multifocal in about $35-40 \%$ of patients $(1,2)$. According to Western guidelines, the treatment of multifocal (non-metastatic) HCC is well codified: transplantation is indicated for HCCs within Milan criteria (2-3 nodules $\leq 30 \mathrm{~mm}$ ), ablation for non-transplantable HCCs within Milan criteria, and chemoembolization for the remaining patients (3). Liver resection has no role. Daily practice is much more complex. The term multinodular HCC encompasses a wide range of scenarios, from oligonodular to diffuse disease, from multicentric to metastatic tumors, that require a case-by-case tailored treatment. We are still far from a real solution and several issues deserve further investigations, including the role of surgery, as highlighted by the recently published Japanese series of liver resections for multiple HCCs (1).

\section{Literature is permissive}

For a long time, few patients with multinodular HCC have been included in surgical series without specific analyses, preventing the possibility to draw any conclusion. In 2008, Ishizawa $e t$ al. reported the first large series with favorable results (126 patients, 5-year survival 58\%) (4). In 2013, Torzilli et al. collected more than 2,000 patients undergoing liver resection for HCC worldwide, including 333 with multiple HCCs (5). BCLC class B (including both large and multinodular HCCs) achieved 57\% 5-year survival. Recently, Fukami et al. analyzed the Japanese nationwide registry: 1,944 patients with 2 or 3 HCCs undergoing surgery were compared with 1,302 patients with similar tumor burden undergoing chemoembolization (1). Resection group had better survival both in the whole series (5-year survival $59 \%$ vs. $42 \%, \mathrm{P}<0.001$ ) and after propensity score matching $(60 \%$ vs. $42 \%, \mathrm{P}<0.001)$, independently from the HCC size $(\leq />30 \mathrm{~mm})$. Superiority of surgery versus chemoembolization in BCLC class B patients has been confirmed by survival benefit analysis (6), one metaanalysis (7), and one randomized trial (8). The latter enrolled 173 patients with multinodular HCCs beyond Milan criteria. Liver resection achieved higher survival than chemoembolization (5-year survival $52 \%$ vs. $18 \%, \mathrm{P}<0.001$ ). The same difference persisted in patients with $>3$ nodules, but only 11 patients were included. According to all those data, the need for an amendment to Western guidelines about the role of liver surgery in multinodular HCC is evident.

\section{A blurred picture}

Evidences are increasing, but the picture is still to focus for several reasons. First, the proportion of patients with multiple HCCs that can benefit from surgery is unclear. In a multiregional longitudinal cohort trial including patients newly diagnosed with HCC, about $14 \%$ of patients with multifocal tumor underwent resection (2). The proportion decreases to $7 \%$ in the Japanese registry, despite an aggressive surgical policy (1). Second, the criteria to select candidates for surgery are lacking. No study 
identified a cut-off value of HCC number beyond which resection is contraindicated. The limit of three nodules has been fixed in analogy with Milan criteria. Even if it is reasonable, results of surgery beyond this threshold are lacking: only 11 patients with $>3$ HCCs were included in the randomized trial by Yin et al. and 22 in the study by Ishizawa et al. $(4,8)$. Donadon et al. recently proposed an "up to 4 and 6" rule. Among 116 patients undergoing resection for multinodular HCC, those with $\leq 4$ tumors, none $>6 \mathrm{~cm}$ in size, had adequate survival (median 52 months), while patients beyond this limit had significantly lower survival (20 months) (9). However, only 7 patients had $>3$ HCCs. Third, the number of nodules per se poorly depicts tumor burden, e.g., a patient having four $2 \mathrm{~cm} \mathrm{HCCs}$ is not comparable with a patient having one $10 \mathrm{~cm}$ HCC plus one $5 \mathrm{~mm}$ lesion. Modern imaging modalities, namely hepatic MRI, identify small nodules otherwise undetectable, increasing the frequency of multinodular HCCs. Further, surgical series included patients operated on for a solitary lesion with intraoperative detection of additional tiny nodules. Are those patients comparable with those having a preoperative diagnosis of multifocal disease? Finally, some studies mixed cirrhotic and non-cirrhotic patients, two separate populations that do not necessarily deserve the same treatment $(10,11)$.

\section{Satellite nodules, multicentric HCC and metastatic HCC}

In multifocal HCC, terminology is crucial. Are we dealing with true multiple HCCs or with satellite nodules? Satellite nodules are macroscopic or microscopic tumor nests situated close to the main tumor $(\leq 20 \mathrm{~mm})$ into the same segment probably due to HCC microscopic vascular invasion. They should not be mis-classified as multifocal HCCs. Much more attention deserves the distinction between multicentric and metastatic HCC: the first is due to the occurrence of multiple synchronous HCCs, while the latter to the onset of a primary tumor with rapid development of intrahepatic metastases (12). Metastatic HCC is expected to have early recurrence because of its aggressiveness, while multicentric HCC is thought to be related to the "field effect" (intrinsic or acquired abnormalities in the liver background) prompting to late relapse. Standards for their distinction are lacking. Pathology criteria have been advanced, but the analysis of clonality is probably the most reliable approach. In a recent analysis by Chianchiano et al. (12), about $20 \%$ of multifocal
HCCs were metastatic and $30 \%$ were multicentric, but $8 \%$ had combined features and $42 \%$ were not classifiable. Different types of multifocality may require different treatment, as recently analyzed for cholangiocarcinoma (13), but univocal and reliable preoperative distinction is needed.

\section{Facing the myth of transplantation}

Most studies compared surgery to chemoembolization, but we should look at a more ambitious standard, i.e., liver transplantation. Is resection an alternative to transplantation in some patients with multiple HCCs? In patients within Milan criteria (2-3 HCCs $\leq 30 \mathrm{~mm}$ ), surgery achieved excellent results: 5 -year survival rate of $71 \%$ in the Fukami et al. series (1) and of $68-75 \%$ according to a recent nomogram (14). Results are quite similar to those after transplantation (75-80\%) (15), even if a conclusive comparison should rely on a longer perspective (at least 10 years of follow-up). Beyond Milan criteria, transplantation has better outcome than resection $(5$-year survival rates $40-55 \%$ vs. $50-75 \%(1,8,15,16)$, but not all centers share such extended indications. Additional studies help elucidating this comparison, as the paper recently published by Pinna et al. (17). Considering the chance of cure of HCC patients (disease-free survival), transplantation is superior to resection within all transplant criteria (Milan or extended ones); considering overall survival, the difference between transplantation and resection is small when oligonodular tumors (2-3 HCCs) are considered, progressively disappearing with the increase of drop-out rate. Survival benefit analyses suggest similar conclusions: transplantation has much higher benefit in Milan-out patients than in Milan-in ones, for whom surgery can guarantee adequate outcome $(6,18)$. A further step forward is mandatory: tumor biology has to be considered. Zaydfudim et al. demonstrated that transplantation is superior to resection in Milan-out patients when transplantation is performed after effective downstaging protocols, while the two treatments have similar results if transplant recipients are not pretreated or fail downstaging (16). In a precision medicine perspective, the number of tumors is no more per se an adequate criterion to define the best treatment.

\section{What about ablation?}

Some considerations about ablation, a further potentially radical treatment, are needed. In multinodular HCC (up to 
3 nodules) ablation is expected to maintain its peculiarities, i.e., excellent short-term results and an HCC sizedependent effectiveness. So far, we can postulate similarity or even superiority of ablation over resection in HCC $\leq 20 \mathrm{~mm}$ distant from major vessels, and a progressively increasing superiority of surgery over ablation with the increase of tumor size (19). Surgery is still the standard for HCCs close to major pedicles. Further, intraoperative ultrasonography is the best staging modality, that in multinodular disease may detect additional lesions in a nonneglectable proportion of patients. The main argument in favor of ablation is its minimal invasiveness, but the diffusion of laparoscopic liver surgery could reduce the distance between the two procedures.

\section{Coming out of the box: a patient-tailored and multimodal solution}

A complex scenario cannot have a simple solution. A strict single-option recommendation, as proposed by Western guidelines, is outdated. In fact, the evaluation of HCC patient should be multiparameter, and not dogmatically based on tumor number. In this scenario, surgery may have different roles. In centers strictly adopting Milan criteria, resection could be scheduled for Milan-in nontransplantable patients (as alternative to ablation) and could be considered for selected patients with oligonodular Milan-out disease, sometimes associated with intraoperative ablation. If extended criteria for transplantation are adopted-a more modern approach in our opinion-liver resection becomes even more relevant. It can be scheduled upfront in some Milan-in patients (planning a salvage transplantation at recurrence) and in selected Milan-out patients as the therapeutic option or as part of an aggressive downstaging protocol.

In conclusion, surgery should be considered in multinodular HCCs, but more robust studies are needed to support clinical practice. The implementation in the decision process of multidisciplinarity and of tumor biology evaluation, including the distinction between multifocal and metastatic HCCs, are the keys to a precision medicine. A personalized multimodal and, sometimes, multistep strategy is needed, combining transplantation, ablation and resection, the latter having different relevant roles.

\section{Acknowledgments}

None.

\section{Footnote}

Conflicts of Interest: The authors have no conflicts of interest to declare.

\section{References}

1. Fukami Y, Kaneoka Y, Maeda A, et al. Liver Resection for Multiple Hepatocellular Carcinomas: A Japanese Nationwide Survey. Ann Surg 2019. [Epub ahead of print].

2. Roayaie S, Jibara G, Tabrizian P, et al. The role of hepatic resection in the treatment of hepatocellular cancer. Hepatology 2015;62:440-51.

3. EASL Clinical Practice Guidelines: Management of hepatocellular carcinoma. J Hepatol 2018;69:182-236.

4. Ishizawa T, Hasegawa K, Aoki T, et al. Neither multiple tumors nor portal hypertension are surgical contraindications for hepatocellular carcinoma. Gastroenterology 2008;134:1908-16.

5. Torzilli G, Belghiti J, Kokudo N, et al. A snapshot of the effective indications and results of surgery for hepatocellular carcinoma in tertiary referral centers: is it adherent to the EASL/AASLD recommendations?: an observational study of the HCC East-West study group. Ann Surg 2013;257:929-37.

6. Vitale A, Burra P, Frigo AC, et al. Survival benefit of liver resection for patients with hepatocellular carcinoma across different Barcelona Clinic Liver Cancer stages: a multicentre study. J Hepatol 2015;62:617-24.

7. Liu W, Zhou JG, Sun Y, et al. Hepatic Resection Improved the Long-Term Survival of Patients with BCLC Stage B Hepatocellular Carcinoma in Asia: a Systematic Review and Meta-Analysis. J Gastrointest Surg 2015;19:1271-80.

8. Yin L, Li H, Li AJ, et al. Partial hepatectomy vs. transcatheter arterial chemoembolization for resectable multiple hepatocellular carcinoma beyond Milan Criteria: a RCT. J Hepatol 2014;61:82-8.

9. Donadon M, Fontana A, Procopio F, et al. Dissecting the multinodular hepatocellular carcinoma subset: is there a survival benefit after hepatectomy? Updates Surg 2019;71:57-66.

10. Vigano L, Lleo A, Aghemo A. Non-alcoholic fatty liver disease, non-alcoholic steatohepatitis, metabolic syndrome and hepatocellular carcinoma-a composite scenario. Hepatobiliary Surg Nutr 2018;7:130-3.

11. Vigano L, Conci S, Cescon M, et al. Liver resection for hepatocellular carcinoma in patients with metabolic syndrome: A multicenter matched analysis with HCV- 
related HCC. J Hepatol 2015;63:93-101.

12. Chianchiano P, Pezhouh MK, Kim A, et al. Distinction of intrahepatic metastasis from multicentric carcinogenesis in multifocal hepatocellular carcinoma using molecular alterations. Hum Pathol 2018;72:127-34.

13. Conci S, Ruzzenente A, Vigano L, et al. Patterns of Distribution of Hepatic Nodules (Single, Satellites or Multifocal) in Intrahepatic Cholangiocarcinoma: Prognostic Impact After Surgery. Ann Surg Oncol 2018;25:3719-27.

14. Torzilli G, Donadon M, Belghiti J, et al. Predicting Individual Survival After Hepatectomy for Hepatocellular Carcinoma: a Novel Nomogram from the "HCC East \& West Study Group". J Gastrointest Surg 2016;20:1154-62.

15. Mazzaferro V, Llovet JM, Miceli R, et al. Predicting survival after liver transplantation in patients with hepatocellular carcinoma beyond the Milan criteria: a retrospective, exploratory analysis. Lancet Oncol

Cite this article as: Viganò L, Costa G, Di Tommaso L. Liver resection for multifocal hepatocellular carcinoma: is it an option? HepatoBiliary Surg Nutr 2019;8(5):530-533. doi: 10.21037/hbsn.2019.05.12
2009;10:35-43.

16. Zaydfudim VM, Vachharajani N, Klintmalm GB, et al. Liver Resection and Transplantation for Patients With Hepatocellular Carcinoma Beyond Milan Criteria. Ann Surg 2016;264:650-8.

17. Pinna AD, Yang T, Mazzaferro V, et al. Liver Transplantation and Hepatic Resection can Achieve Cure for Hepatocellular Carcinoma. Ann Surg 2018;268:868-75.

18. Vitale A, Morales RR, Zanus G, et al. Barcelona Clinic Liver Cancer staging and transplant survival benefit for patients with hepatocellular carcinoma: a multicentre, cohort study. Lancet Oncol 2011;12:654-62.

19. Vigano L, Laurenzi A, Solbiati L, et al. Open Liver Resection, Laparoscopic Liver Resection, and Percutaneous Thermal Ablation for Patients with Solitary Small Hepatocellular Carcinoma $(</=30 \mathrm{~mm})$ : Review of the Literature and Proposal for a Therapeutic Strategy. Dig Surg 2018;35:359-71. 